\title{
Supporting Information for: Two Phases of Monolayer Tantalum Sulfide on Au(111)
}

Daniela Dombrowski, ${ }^{,+,+\ddagger}$ Abdus Samad, " Clifford Murray, ${ }^{\S}$ Marin Petrović,

Pascal Ewen, ${ }^{\dagger}$ Thomas Michely, ${ }^{\S}$ Marko Kralj, I Udo Schwingenschlögl, $₫$ and Carsten Busse ${ }^{\dagger, \ddagger}$

$\dagger$ Institut für Materialphysik, Westfälische Wilhelms-Universität Münster, 48149 Münster, Germany

$\ddagger$ Department Physik, Universität Siegen, 57068 Siegen, Germany

TKing Abdullah University of Science and Technology, Physical Science and Engineering Division, 23955 Thuwal, Saudi Arabia

§II. Physikalisches Institut, Universität zu Köln, 50937 Köln, Germany

$\|$ Center of Excellence for Advanced Materials and Sensing Devices, Institute of Physics, 10000 Zagreb, Croatia

E-mail: dombrowski@wwu.de 


\section{ARPES Data of Clean Au(111)}

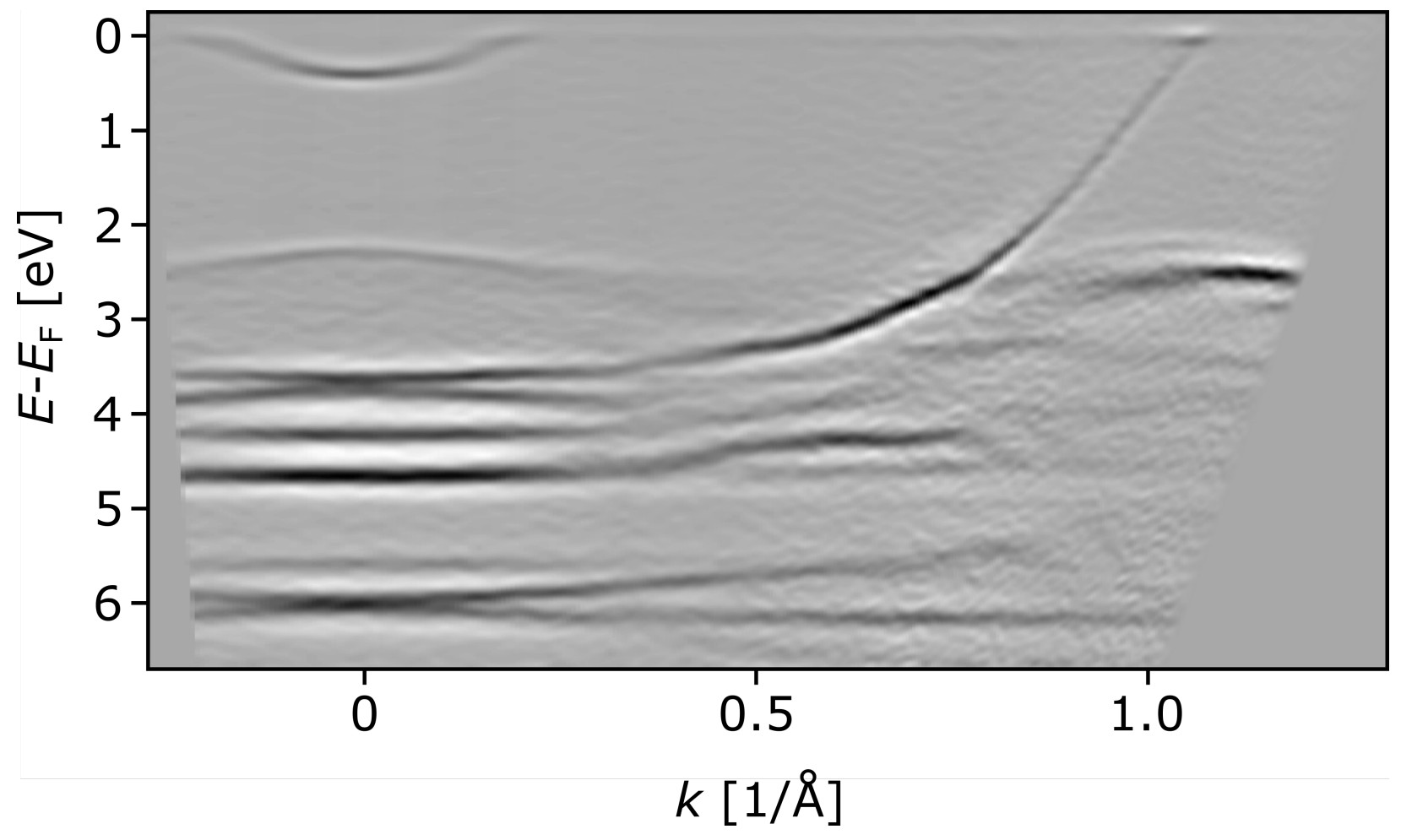

Figure S1: ARPES data of the clean $\mathrm{Au}(111)$ surface (reference spectrum) after an LucyRichardson (LR)-deconvolution to improve signal-to-noise ratio, ${ }^{22}$ and forming the second derivative with respect to energy to enhance features. Visible are the Shockley surface state at $k=0 \AA^{-1}$ and the projected bulk sp-band, which crosses the Fermi level at $k \approx 1 \AA^{-1}$, as well as several d-bands of gold. 


\section{ARPES Data of the Shifted SS of $\beta$-Phase on Au(111)}

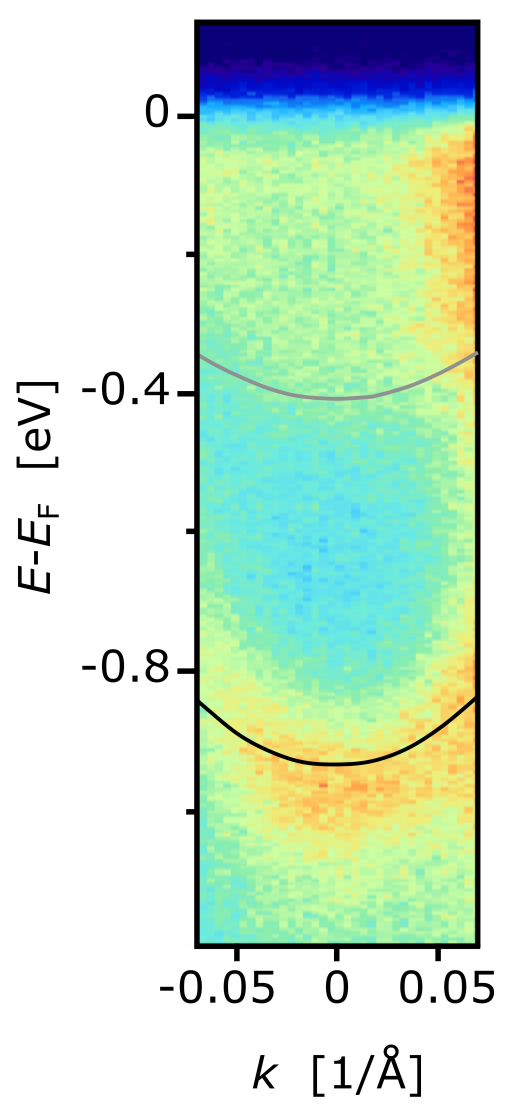

Figure S2: ARPES data of $\beta$-phase on $\mathrm{Au}(111)$ with total coverage of $\Theta_{\beta} \approx 0.64$ ML showing two electron like bands at the $\Gamma$ point, which we attribute to the SS of clean $\mathrm{Au}(111)$ and to the shifted SS of $\beta$-phase/Au(111). For comparison: The parabolic lines superimposed to the ARPES data are the fit results from clean $\mathrm{Au}(111)$ (gray line, extracted from Figure S1) and from $\beta$-phase $/ \mathrm{Au}(111)$ with $\Theta_{\beta} \approx 0.86 \mathrm{ML}$ (black line, data shown in the main text). 


\section{DFT Calculation of Free-Standing TaS}

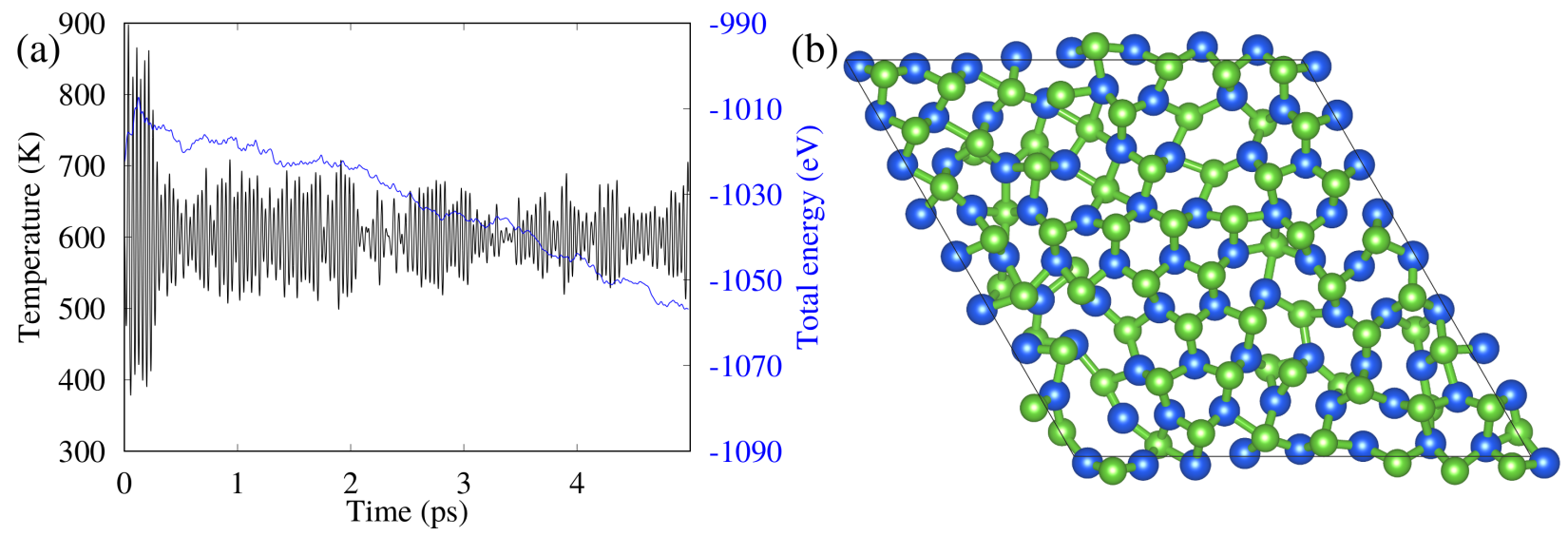

Figure S3: Ab initio molecular dynamics (MD) simulations for free-standing TaS at an average temperature of $600 \mathrm{~K}$. (a) Evolution of temperature and total energy over 5 ps with a time step of 1 fs. (b) Structure after 5 ps (Ta atoms: blue, S atoms: green) 